\title{
INCORPORATING SHAPE-CHANGING FOOD MATERIALS INTO EVERYDAY CULINARY PRACTICES
}

\author{
Guidelines Informed by Participatory Sessions with Chefs Involving Edible pH-responsive Origami
}

\section{Structures}

Elzelinde, E., Van Doleweerd Industrial Design, Eindhoven University of Technology, The Netherlands. And Upprinting Food, Eindhoven, The Netherlands.

\author{
Ferran, F., Altarriba Bertran
}

Social Emotional Technology Lab, UC

Santa Cruz, United States.

\author{
Miguel, M., Bruns Alonso \\ Industrial Design, Eindhoven \\ University of Technology, The \\ Netherlands.
}

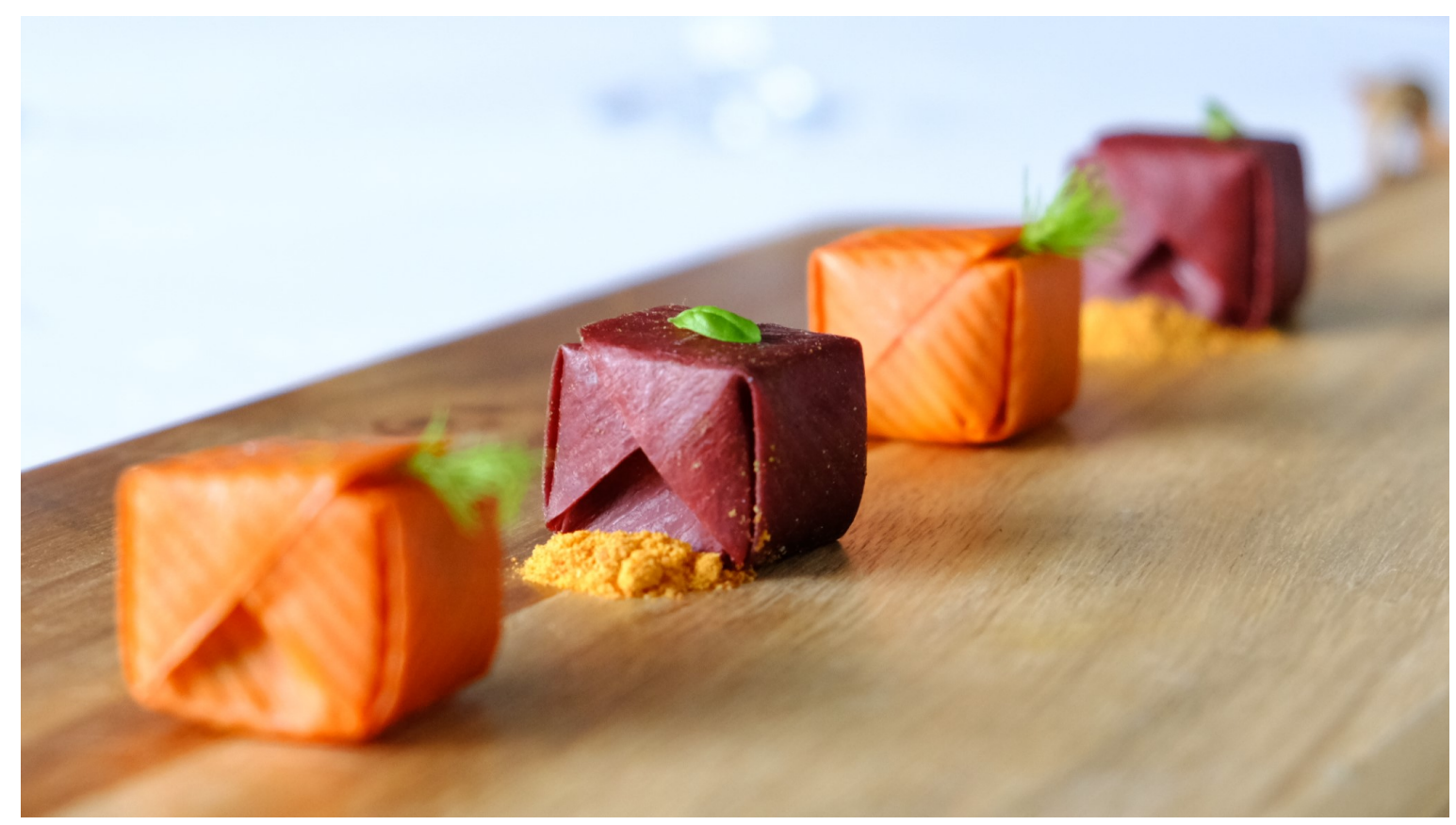

Figure 1: Carrot and tomato-based edible paper with a 3D printed striped chitosan pattern, folded in cubes which reveal their content when served with a low pH-liquid.

\begin{abstract}
In recent studies researchers have developed compelling and exciting shape-changing food (SCF) materials. Unfortunately, the adoption of SCFs in culinary practice is still complex. We present a research-through-design exploration of a novel SCF material that can be developed in restaurant kitchens using everyday kitchen appliances with the only addition of a commercial desktop 3D food printer. Our lightweight process involves the preparation of edible
\end{abstract}

This work is licensed under a Creative Commons

Attribution-NonCommercial-ShareAlike International 4.0 License.

TEI '22, February 13-16, 2022, Daejeon, Republic of Korea

(C) 2022 Copyright held by the owner/author(s).

ACM ISBN 978-1-4503-9147-4/22/02.

https://doi.org/10.1145/3490149.3501315 paper, which can be folded into origami shapes and is actuated through the addition of an acidic liquid. We facilitated participatory sessions with five chefs in their restaurants and evaluated our material. Our qualitative study contributes design guidelines on how to incorporate SCFs into chefs' everyday culinary practices. Building on the chefs' perspectives of our material and its culinary potential we conclude with a speculation of how SCF materials could enable novel multi-sensory and interactive food experiences.

\section{CCS CONCEPTS}

- Human-centered computing $\rightarrow$ Interaction design; Systems and tools for interaction design.

\section{KEYWORDS}

Human-Food Interaction, Digital Gastronomy, Shape-changing Materials, Origami, 3D Food Printing 
ACM Reference Format:

Elzelinde, E., Van Doleweerd, Ferran, F., Altarriba Bertran, and Miguel, M., Bruns Alonso. 2022. INCORPORATING SHAPE-CHANGING FOOD MATERIALS INTO EVERYDAY CULINARY PRACTICES: Guidelines Informed by Participatory Sessions with Chefs Involving Edible $\mathrm{pH}$-responsive Origami Structures. In Sixteenth International Conference on Tangible, Embedded, and Embodied Interaction (TEI '22), February 13-16, 2022, Daejeon, Republic of Korea. ACM, New York, NY, USA, 14 pages. https://doi.org/10.1145/3490149. 3501315

\section{INTRODUCTION}

When going out for dinner in a restaurant, not only the taste, but also the entire multi-sensory experience is important [24]. The visual aesthetics of the food, the sound ambiance, the shape and texture of cutlery, the way we put food in our mouth, or even the social interactions that take place throughout the dinner have an impact on both our taste perception and on our holistic experience of the meal [24]. Consequently, to craft food experiences that are compelling, chefs use all sorts of strategies and techniques. For example, molecular gastronomy techniques (inspired by elBulli, arguably the most influential avantgarde cuisine restaurant in the last decades [20]) allow chefs to create a surprising mouthfeel to their food. More recently, chefs have started to adopt digital technologies like 3D printing to create complex food designs [38].

In Human-Computer Interaction (HCI), there is a growing body of works that target the intersection of food, technology, and human interaction [1]. The so-called space of Human-Food Interaction (HFI) is an umbrella for research that explores ways in which technology could ease, augment, make more accessible, or enrich our interactions with, trough, and around food [1]. Within HFI, there are efforts to support increasingly interesting gastronomic futures. Here we focus on a subset of that body of research: works that look at the potential of augmenting foods with shape-changing properties. Inspired by previous efforts in shape-changing materials for interaction design (e.g., [25, 27, 34]), HFI researchers are exploring how to craft shape-changing food (SCF) materials that provide chefs with new ways of surprising diners. For example, Wang et al.'s Transformative Appetite is a technique for producing pasta-like foods that change shape when cooked in water [30].

Importantly, most of the research done in the space of SCF materials has so far been technically focused; little efforts have been put to exploring how it might function outside of the lab, in real gastronomy scenarios. Generally, research outcomes in this space are future-oriented speculations that are envisioned to be used in futuristic dining experiences; in the few cases where the produced shape-changing materials are ready to be eaten today, little effort has been devoted to ensuring that those materials respond to the needs of diners or to support chefs to integrate them in their own creative processes. Our research explores how to fill this gap.

In this paper, we developed a SCF material using ordinary kitchen equipment, ingredients, and processes, with the only addition of a commercial desktop 3D food printer (fig. 1). Chefs can recreate this material and use it in their own practice, to create novel dining experiences. In addition to a step-by-step instruction of a flexible SCF material that chefs can use as a basis to create their own SCFs, we conducted participatory sessions with chefs in their restaurants. Through our qualitative evaluation, we investigated their perspectives on the potential of our shape-changing material in gastronomy, and the uses they might find for it in their culinary practice. We contribute a set of design guidelines on how to incorporate SCFs into chefs' everyday culinary practices. Finally, building on our conversations with chefs, we speculate how our material could give rise to increasingly multi-sensory, interactive, and playful food experiences for diners, both of which have recently been called for in HFI $[4,19]$ and gastronomy design [3]. Overall, the aim of our study is to reduce the gap between SCFs research and culinary practice by empowering chefs with a new technique to enrich their creative possibilities and inspiring other designers in HFI interested in creating similar and increasingly chef-friendly tools and materials for interactive gastronomy design.

\section{RELATED WORK}

Various projects have explored how novel technologies can contribute to innovations in the domain of Human-Food Interaction (HFI). In particular, using digital manufacturing and drawing inspiration from material science to create edible shape-changing materials has gained popularity. In this section we first provide an overview of the design and research space of HFI, the area where we position our work. Following, we narrow down to the subset of HFI research our work relates to directly: we discuss projects that incorporate digital manufacturing and food science to transition from SCFs to the concept of digital gastronomy. Finally, we look at other relevant works within Interaction Design, Food Design and Gastronomy to review a series of concepts that inspired our work.

\subsection{Human-Food Interaction}

HFI is an emerging research area within Human-Computer Interaction that explores the intersection between food, technology, and human interaction. Because food practices are extremely diverse, HFI encompasses a variety of works targeting myriad areas of the food chain, including sourcing food materials (e.g., growing or buying them), storing and wasting food, producing elaborated foods (e.g., by cooking or through industrial processes), eating and drinking, tracking food practices (e.g., monitoring food intake), or speculating about alternative food futures [1].

HFI is not only diverse in terms of the areas of food practices it studies; it also encompasses radically different (sometimes even opposing) approaches to the interplay between humans and technology (e.g., technology automation vs. human empowerment), and targets a number of different research agendas (e.g., enriching dining experiences multi-sensorially, making food production easier and safer and supporting social interaction during meals). Such diversity in domains of implementation, approaches to humantechnology integration, and research agendas constitutes a field that, despite recent efforts to structure it (e.g., $[1,5])$, is still quite underdefined.

\subsection{From Shape-Changing Food Design to Digital Gastronomy}

Within food production oriented HFI, there is a body of works that explore the potential of computational tools to support chefs in creating novel dining experiences involving shape-changing foods. We see research that focuses on different approaches, materials, 
and target groups within this space. In Morphlour grooved patterns in flour-based pasta result in a transformation mechanism that triggers a shape change by dehydration through baking and hydration through cooking [28]. In Transformative Appetite, pasta-like gelatin-cellulose based films are created and transformed by hydration through cooking. To show the possible implementation of the interactive food, dishes with this material were co-developed with one chef [30]. In both examples, the advantage of flat-packaged food is emphasized, which also shows the intention of applying it as a finished product.

Taking a more material-centered approach, Organic primitives explores $\mathrm{pH}$-reactive materials in a variety of contexts, one of them being 'edible'. With the use of chitosan, pasta was created where shape transformations were defined by patterning specific areas, providing possibilities for chefs to alter the shape of the pasta to enable different levels of flavor retention or sensory augmentation [16]. In Natto Cells, a biofilm is created for shape-changing interfaces. This material is a living, food-save organism and can potentially be used in actuated food [34]. Finally, Digital Konditorei presents a modular silicon mold, supporting flexibility for diners and chefs' requirements, and providing the possibility for chefs to program the taste of food [37]. The overarching concept of such personalized or interactive food experiences have also been defined as Digital Gastronomy [18]. It suggests that digital fabrication techniques can be implemented in the traditional kitchen, allowing personalization in the design parameters by the chefs. The team working on Digital Gastronomy included amongst others a professional chef and a chemist who is also a professionally trained cook [18]. However, beyond oftentimes the involvement of a single chef, the projects described above did not explore how their technology could be extended to and appropriated by a wider audience.

\subsection{The Intersection of Interaction Design, Food Design, and Gastronomy}

In addition to the use of computational tools to create intricate food compositions, we also see relevant innovations in gastronomic experiences that focus on other strategies. Within HFI, a body of works explores how multi-sensory stimuli can have an impact on our food experiences-both on the experience as a whole and on taste perception in particular. In this space, often referred to as $\mathrm{Mul}$ tisensory HFI [1] or Gastrophysics [24], researchers use cross-modal psychology techniques to investigate how the different components of a meal can be leveraged purposefully to enrich a dining experience. Examples are how the color of a plate might influence the perceived taste of the food it contains [21], how the ambient sound of a restaurant influences the pace at which a diner eats [26], or how the texture and weight of cutlery affect how the food it carries is perceived [29], among others.

Another relevant line of works in food-related interaction design are those that explore novel and increasingly interactive ways of eating. Both in food design (e.g., [2, 3]) and in technology research (e.g., $[4,19]$ ), we see works that explore how interactions with, through and around food could be more playful, explorative, surprising, or social. Interestingly, in this space, we also see works that advocate for increasingly participatory approaches to human-food interaction design (e.g., [31]), i.e., HFI design and research that takes place in-the-wild [22] and engages multiple stakeholders as creative partners. From these works, we learn that there is a need for exploring how food materials can help chefs in crafting increasingly multi-sensory, interactive, and playful eating experiences. We are also inspired by how some of these works involved chefs and other relevant stakeholders as co-designers, to reflect their preferences and facilitate a design process that was more situated and contextually sound. We believe that these premises are important when it comes to envisioning new ways of augmenting food materials with shape-changing properties, and we use them as inspiration in our work.

\section{METHOD AND DESIGN PROCESS}

Our research followed a multi-method approach to open new avenues in SCF design and digital gastronomy, inspired by increasingly multi-sensory, playful and participatory approaches to gastronomy design. First, we developed a material probe by means of research-through-design [36], involving an iterative hands-on design process, in which various material samples were created and tested using off-the-shelf materials, basic kitchen equipment and the WiibooxSweetin 3D food printer. Subsequently, we held a participatory session with chefs, using the material probe as a source of inspiration. The participatory sessions were followed by interviews, which we transcribed and analyzed by means of inductive thematic analysis [8]. The thematic analysis resulted in twelve themes, which were grouped into four clusters. Based on the outcomes of our analysis we derived four guidelines on how chefs can incorporate SCFs into their everyday culinary practice. Finally, we speculate about opportunities for design, building on our experiences with the domains of HFI and shape-changing interface research. Overall, we contribute a new and inherently chef centric SCF material, together with an understanding of how it might open new, increasingly playful and interactive avenues for gastronomy design.

\subsection{Developing an Interactive Origami-Inspired Food Material Probe}

The goal of this phase of the project was to create edible, foldable, and shape-changing paper that could be produced in a kitchen context. Folding food is a traditional technique that is often used with dough materials for making products like pastry, pasta, or dumplings. Inspired by those ancient culinary practices, we set out to investigate how to create complex origami shapes with edible paper. Origami originates as a Japanese art form, which is now widely implemented as a form-giving tool, appropriate for ideation techniques. It is applied in product design [10], architecture [12], fashion [9] and given its complex mechanical qualities [6], origami and its variation kirigami (which also involves cutting the paper) have inspired various researchers in $\mathrm{HCI}$ to use it in the context of shape-changing interfaces [e.g., 13, 35]. However, the use of origami in food projects is scarce, although it can be compared to traditional cooking techniques involving ravioli, dumplings, sushi, or marzipan pie decorations.

In this project, we developed origami structures with edible paper, initially based on carrots and we explored how these shapes 


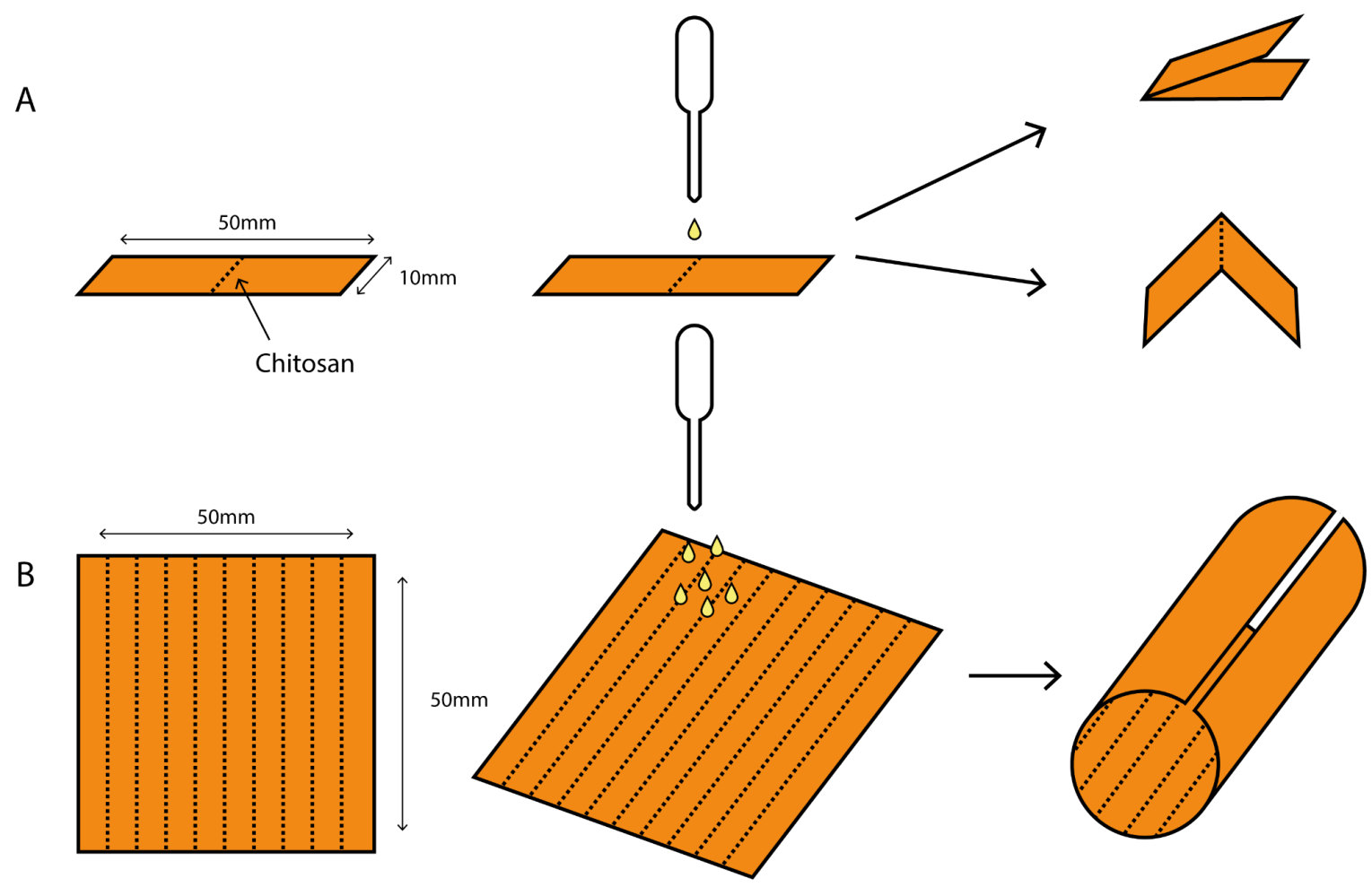

Figure 2: Carrot paper in combination with chitosan solution in strips and squares, reacting with lemon-juice.

can fold or unfold on the plate using low $\mathrm{pH}$-liquids such as lemonjuice. $\mathrm{pH}$-reactivity with chitosan was developed in combination with the edible paper to enable controlled shape-change. In this section, we first describe the research that led to the morphing material. Secondly, we illustrate the interactive properties of the material when it is combined with the edible paper. Following, we show how we optimized the 3D-printing technology which was used to depose the morphing material onto the edible paper. To close, we present the material probe that was shared with the chefs in a participatory session.

3.1.1 Development of the Responsive Shapes Based on a Chitosan Solution. Kan et. al. [16] describe how the polysaccharide chitosan can be used to create a shape-changing material. Chitin is a biopolymer that is typically isolated from the exoskeleton of crustaceans (e.g., shrimp, crab or prawn), a by-product of marine food processing (as alternative it can be isolated from mushrooms or insects). From the chitin, acetyl-groups are removed, leaving a complete amino group behind resulting in the soluble chitosan. Chitosan is often used for edible coatings, e.g., to extend the shelf life of fruit and vegetables [23]. Chitosan can swell in low $\mathrm{pH}$ conditions (in our research we use lemon-juice to create this low $\mathrm{pH}$ condition) due to crosslinking reaction of the amino groups (i.e., $\mathrm{NH} 2$ becomes $\mathrm{NH} 3+$ ). This reaction is reversible by increasing the $\mathrm{pH}$ of the solution [33]. An acidic solution has to be used to dissolve chitosan [7] for which acetic acid is typically used. For this research vinegar was chosen, as the product will be prepared in a restaurant kitchen. Commercially available vinegar contains around $4-8 \%$ of acetic acid.
Because in our experiment we used vinegar instead of acetic acid, we had to modify the material composition presented by Kan et al. [16]. To test the different compositions, thin films were created by drying the solution in a dehydrator, which were cut into small strips. The small strips were compared on their reactivity by pipetting lemon juice onto the centerline. This resulted in chitosan films with $5 \%$ weight per volume $(\mathrm{w} / \mathrm{v})$ chitosan powder, dissolved in a solution of $50 \%$ vinegar and $50 \%$ water.

3.1.2 Development of the Edible Paper. Xuerong et al. [32] proposed a method to create edible paper in a laboratory environment, their preparation method had to be adjusted to a kitchen context. A kitchen mixer was used instead of a laboratory blender and no degassing step was executed. With a tissue masher, the particles of the puree can become smaller with the same cooking time and through degassing, air-bubbles are removed. Due to these changes, a longer cooking time was required to create a similarly smooth puree, which also results in more water absorbance and an extended dehydration process. The final composition of the morphing edible paper had to meet three conditions: it had to be foldable; it had to change its shape after application of a low $\mathrm{pH}$ liquid in combination with the chitosan; and the paper should not absorb the chitosan-solution easily, to preserve the optimal chitosan-solution. Based on Xuerong et al [32] the basic paper was created using the following ingredients: Carrots, $\mathrm{NaHCO}_{3}$, starch, glycerol, sodium alginate, agar, CMC-Na. Various experiments were executed with increased concentration of the individual ingredients to evaluate their effect on the dried edible paper sheets, both on the foldability 


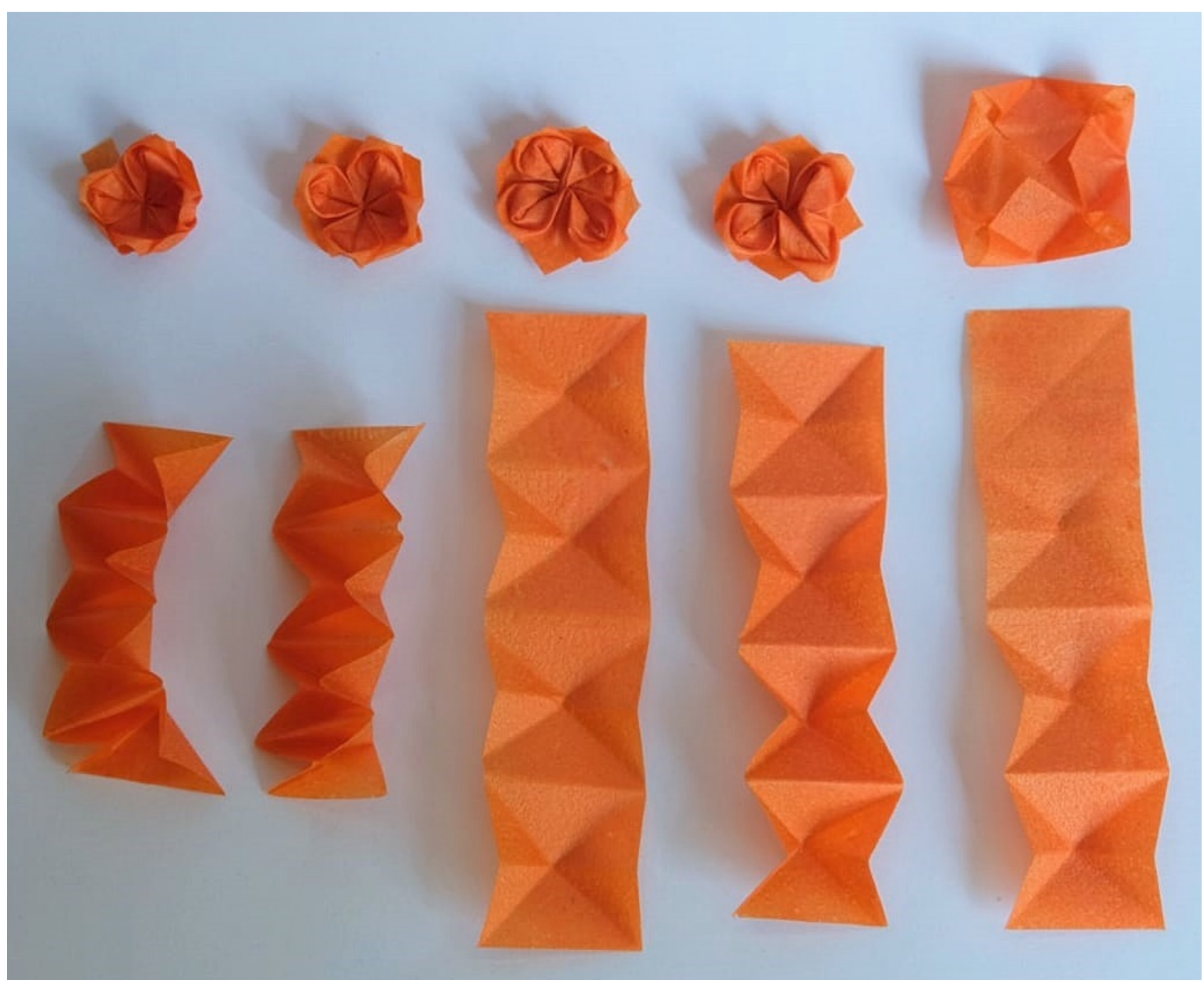

Figure 3: Origami structures to evaluate the ability of the edible paper to hold its folded shape.

of the edible paper sheets and on the reaction with the lemon-juice in combination with the chitosan.

To evaluate the reactivity, the edible paper sheets were cut into squares of $50 \times 50 \mathrm{~mm}$ and strips of $10 \times 50 \mathrm{~mm}$. The chitosan solution was deposited on the centerline of the strips and in lines, spaced $2 \mathrm{~mm}$ apart on the square pieces of paper. The reactions were limited, only the paper sheets with the increased concentration of methylcellulose and the paper sheets with the increased concentration of alginate showed two bending directions or turned into a tubular cannolo shape when the lemon juice was added (fig. 2). To evaluate their foldability, all sheets were folded in two origami structures and evaluated on the ability to hold its folded shape. Both the paper with the increased concentration of methylcellulose and the alginate lacked the ability to hold its shape when folded (fig. 3). A balance between reactivity and foldability was needed, which resulted in the final puree following the composition and ingredients of Xuerong et al [32] with an additional $1 \%$ of alginate.

In the final composition, several flavors were created by substituting the base with other ingredients. Using a puree offers the possibility of taking a sustainable approach when creating the material, because the shape or structure (ripeness) of the food does not influence the final appearance. Several experiments were done to support chefs in their creative process during the evaluation. First, dried tomato powder was added to the carrots, creating a different taste and feeling of the paper. In a second experiment, overly fermented plums were used. When fermenting fruit or vegetables too long, they lose structure and will get mushy and sour. The same counts for overly fermented kohlrabi as a third, and overly fermented kohlrabi with mustard as a fourth experiment (fig. 4).

3.1.3 Shape and Print Developments. With the final edible paper composition, new tests were done to determine the optimal concentration of chitosan. A higher $(>6 \% \mathrm{w} / \mathrm{v})$ chitosan concentration, results in a more viscous solution, which leads to a minimal visible reaction when dried into a film. Conversely, when using a lower concentration of chitosan in percentage $\mathrm{w} / \mathrm{v}$, the equilibrium degree of swelling increases, resulting in more $\mathrm{pH}$-sensitive films in reaction to the lemon juice [33]. For the final chitosan solution, a concentration of $6 \% \mathrm{w} / \mathrm{v}$ chitosan was used. 3D-printing supported the deposition of multiple layers of the chitosan-solution exactly on top of each other (fig. 5). Due to its low viscosity, it was required to dry the first layer of solution before printing the second layer. 


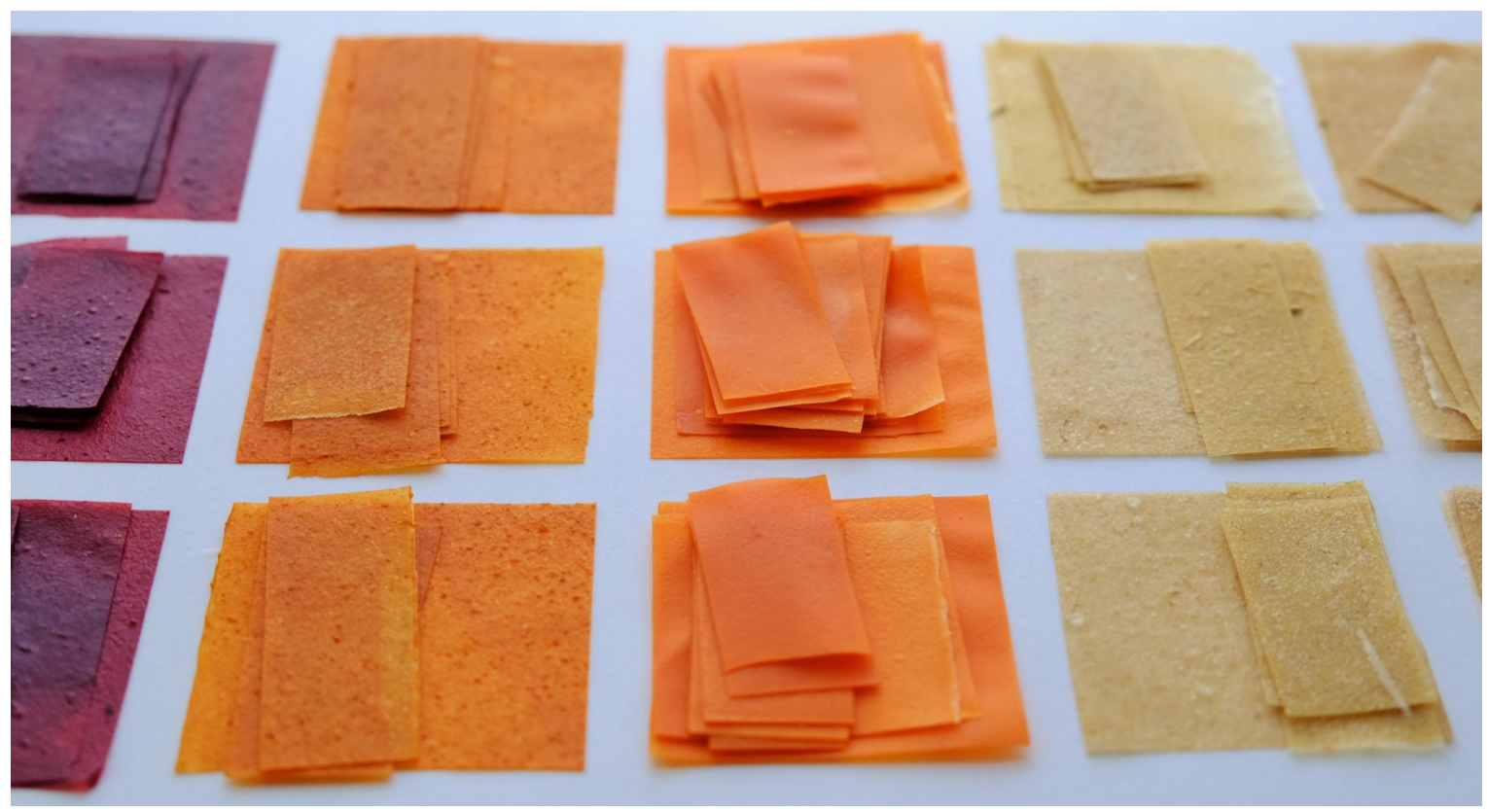

Figure 4: Five different flavors: fermented plums; carrots with tomato powder; carrots; fermented kohlrabi with mustard; fermented kohlrabi.

Based on previous work involving folded structures $[15,17]$, we assumed that the shape-transformation would take place by printing the fold lines of the structures with the chitosan. However, after printing the fold lines and folding the structures, the shape did not unfold in reaction to a low $\mathrm{pH}$ solution. It appeared that a constant line pattern not only worked best for a tubular-shape like cannoli, but also for the unfolding of a cube and even the transformation of a star-shape, shown in figure 6 . The origami structures were created with $100 \mathrm{~mm} \times 100 \mathrm{~mm}$ sheets of printed edible paper, lemon juice was poured on top. While with a single layer of chitosan solution printed, no reaction was shown, with a double layer, a slow but clearly visible reaction occurred.

\subsection{Participatory sessions with Chefs: Exploring the Use and Potential of Our New Material}

To understand how chefs might use this new material in their culinary practices, an evaluation was set-up to study how they perceived and would use the edible, foldable paper. A material probe was employed as a starting point for a discussion in a series of participatory sessions. In these sessions chefs tasted, played with, and otherwise experimented with the material to understand what was interesting about it or how they could use it in their practice. Below we describe the process in detail and the outcomes of the interviews after the participatory sessions.

3.2.1 Participants. A total of five chefs (numbered 1-5) participated in this study. The chefs were recruited through social media, after their interest in the first authors' work on digital manufacturing of sustainable food. All chefs have experience working in different fine-dining restaurants in which aesthetic plating is important. Two chefs had experience in working with a 3D-food printer. Four chefs (numbered 1-4) went through the process in pairs together with the researcher. One chef (numbered 5) received a material probe by mail, together with pictures, videos and a file which guided the chef through the process step by step.

- Chef 1: Australian. Has worked in various restaurants, among which an Australian one Michelin starred restaurant. He currently works in a Dutch restaurant which focuses on serving good food while reducing food waste (from e.g., farmers and supermarkets), as a sous-chef. He has experience with 3D food printing.

- Chef 2: Greek. Has worked worldwide in various Michelin starred restaurants, amongst which a two starred Danish restaurant and a three starred British restaurant as well as freelance chef. He currently works in the same restaurant as Chef 1 as a head-chef. He frequently experiments with fermentation techniques and has experience with 3D food printing.

- Chef 3: Dutch. Has worked in various Michelin starred restaurant. He currently is the owner and head-chef of his own fine-dining Dutch restaurant. He is very creative and changes the menu every month. He tries to make optimal use of the ingredients which he orders for the menu of his restaurant.

- Chef 4: Spanish. Has worked internationally in many restaurants, among which a Dutch three Michelin starred restaurant. He currently works in the same restaurant as Chef 3 as a sous-chef. He enjoys developing himself in both savory and pastry and techniques such as fermentation. 


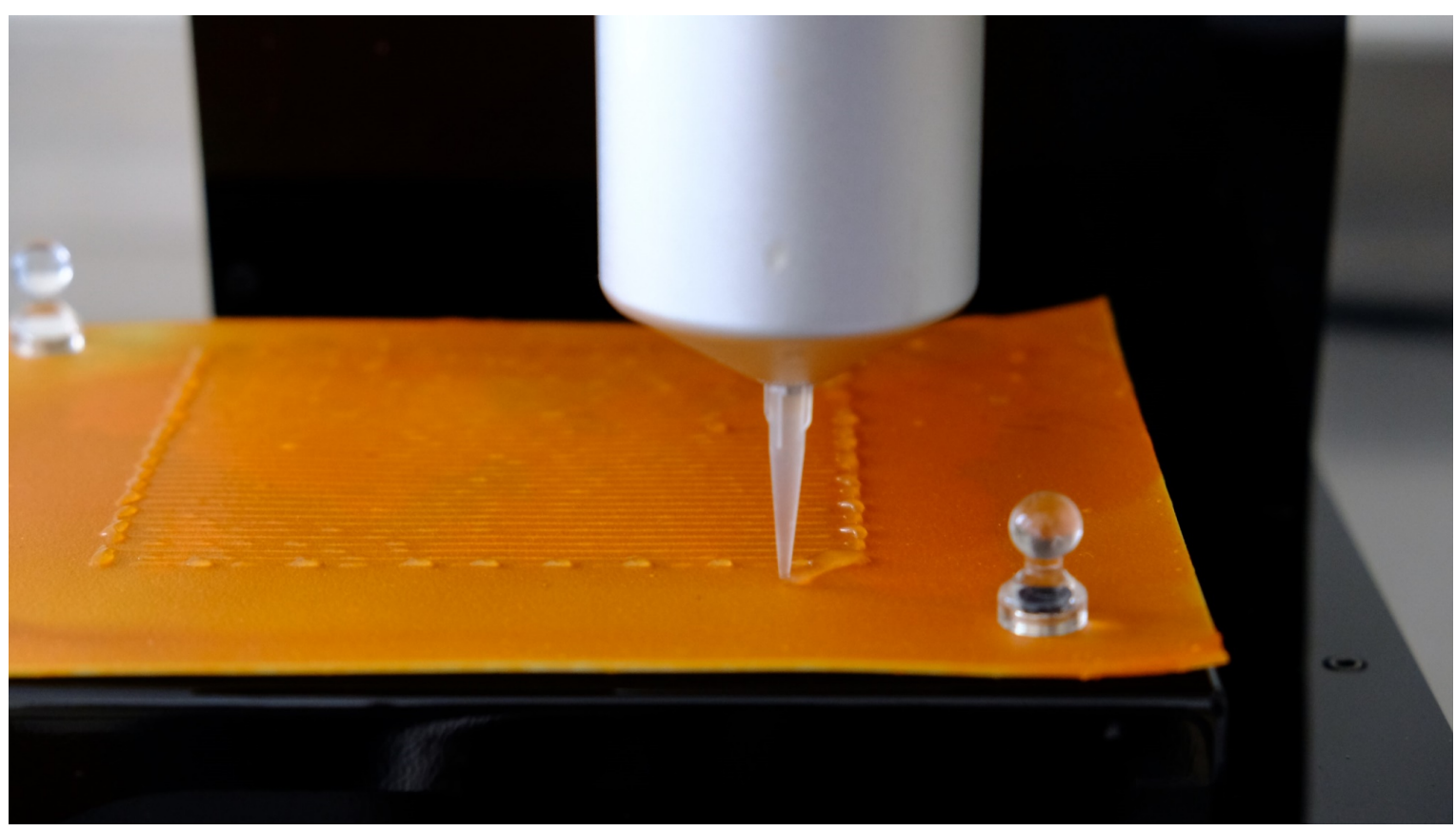

Figure 5: Chitosan is printed with a line-pattern on top of the carrot paper.

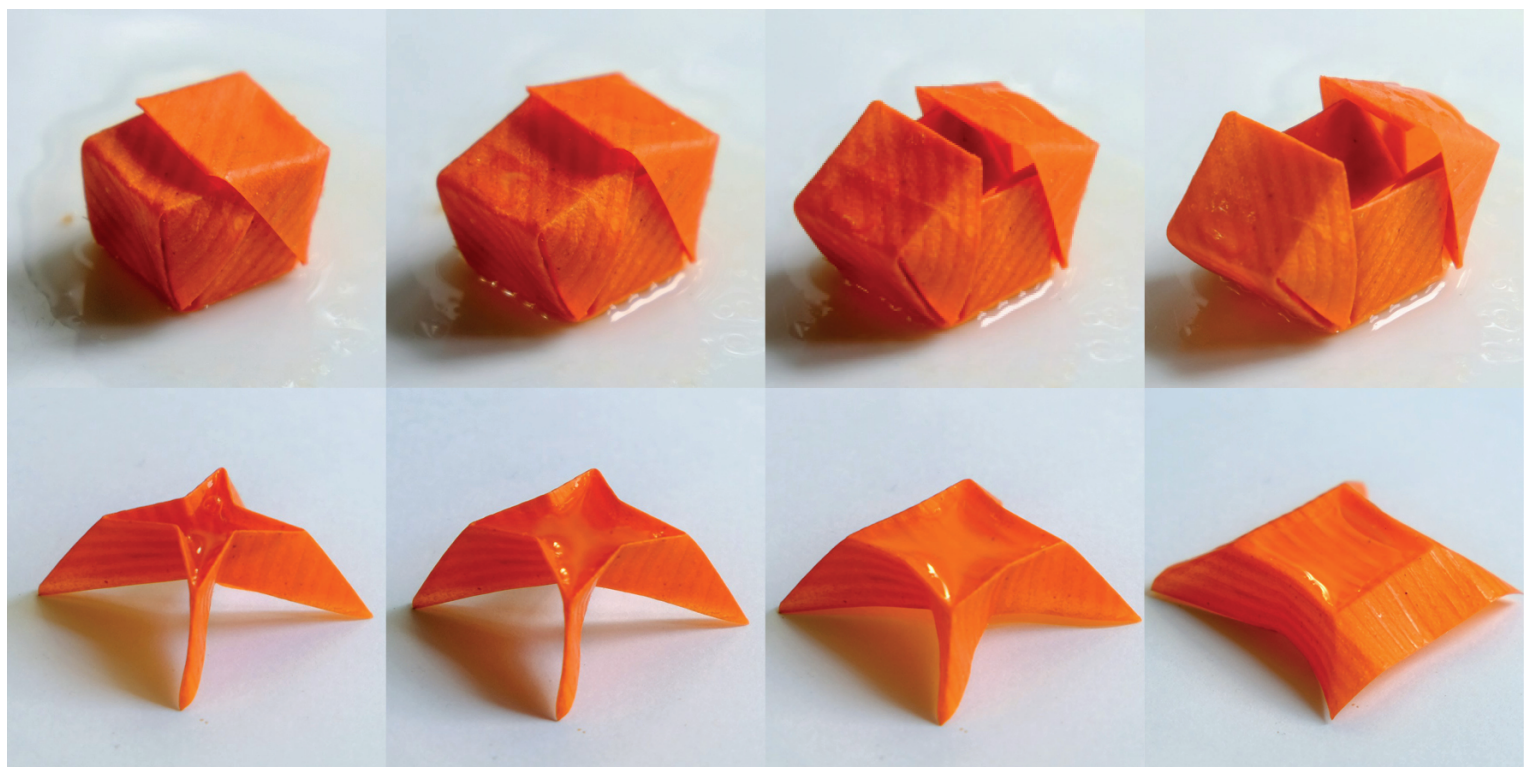

Figure 6: The transformation of an origami cube and a star-shape.

- Chef 5: Brazilian. Has worked as chef for over 20 years, mostly in Brazil and Ireland in both cafes, fine dining restaurants and as owner of her own café and catering company. She currently is chef of a café in Ireland. She has a passion for pastry and works on a project to reduce surplus food by making gelato.
3.2.2 Set-up of the Participatory sessions. The goal of the sessions was to gain insights on how chefs could appropriate the material in their kitchen. Therefore, the sessions were divided into three sections with a clear focus to gain unbiased feedback on (1) the flavor and preparation method of the paper, (2) the foldability, and (3) the shape-transformation, each with its own materials, explanation, and visualization. Each session lasted approximately two hours. 
1. Tasting: Chefs were first invited to taste and evaluate the five different flavors of paper which were developed in advance: carrot, carrot with tomato powder, fermented plums, fermented kohlrabi and fermented kohlrabi with mustard. The researcher discussed the ingredients, preparation method (fig. 7) and chefs' familiarity with both. The material probe for this section consisted of edible paper in five different flavors (fig. 4, fig. 9).

2. Folding: Secondly, chefs experienced folding an origami structure themselves. First the researcher explained how to fold an origami structure with normal paper (fig. 8) and subsequently asked chefs to do the same with the edible paper. The material probe for this section consisted of paper to test the folding experience: two sheets $160 \times 40 \mathrm{~mm}$ of normal white paper to practice how to fold the origami structure; one sheet $160 \times 40 \mathrm{~mm}$ of carrot-paper to fold an edible origami structure (fig. 9).

3. Transforming: Finally, the chefs were introduced to the paper's shape-changing properties. The researcher showed the paper with the printed chitosan lines and invited the chefs to place the paper in lemon juice. Videos were shown of more complex and slower transformations to initiate a discussion of possible ways of implementing the edible paper in the chefs' culinary creations. The material probe for this section consisted of carrot-paper with printed chitosan to test the changeability: one large sheet $100 \times 100 \mathrm{~mm}$; three small sheets $(30 \times 15 \mathrm{~mm})$ to test the transforming cannoli; one folded star structure (fig. 6, fig. 9) and lemon-juice.

3.2.3 Data collection and analysis. The sessions were recorded, and the data was transcribed. Pictures of the process were made by the researcher. Interview data was analyzed through inductive thematic analysis [8]. First, the initial codes were set by the lead author after which ten initial themes emerged. The two other authors participated in a final clustering session: one expert in (transformative) materials research and another expert in human-food interaction design and research. Both researchers received the initial ten themes and checked 35 quotes to facilitate a discussion on how and if the ten initial themes covered the data. The formation of the final themes was the outcome of that discussion.

\section{OUTCOMES OF THE PARTICIPATORY SESSION}

In each section of the participatory session, chefs were asked to share the way they experienced the materials and process. They were also asked if they were familiar with similar processes and materials or ingredients, and if they could envision implementing this in their own work. We summarize the results of the analyses of the sessions below.

\subsection{Tasting}

The chefs used different senses to shape their opinions on the edible paper. Chef 2 focused heavily on the smell and smelled all different flavors first (fig. 10); he perceived the five flavors differently, regarding the way they smelled. Chefs 3 and 4 focused mostly on the mouthfeel of the paper. They evaluated characteristics of the mouthfeel accordingly: "it is less soggy" (Chef 3) and "it melts well"
(Chef 4). For the other chefs taste itself was more important, as the fermented vegetables were quite sour, salty, and strong in flavor. Some implementation ideas also build on this strong acidic flavor of the paper, four chefs mentioned implementing the paper on its own and two chefs considered multiple times how it could be hidden between ingredients as an unexpected flavor component.

All chefs showed a clear interest in both the preparation process and ingredients used. When certain processes or ingredients were familiar to them, they often made suggestions and provided examples related to the sensory perception. Also, they all suggested alternative ingredients and preparation methods to create different flavors. We saw a clear difference in opinion when using specific methods and ingredients, e.g., chefs 1, 3 and 4 used Texturas (an ingredient often used in molecular cuisine to change the texture of food [11]) in their daily work, while others did not (Chef 5) or preferred not to (Chef 2).

\subsection{Folding}

The folding process (as shown in fig. 8, fig. 10) was perceived to be difficult, but all five chefs were open to learn it. Four chefs suggested to slightly increase the thickness of the paper, to make the folding lines more visible. Three chefs mentioned that the (shape-changing) food materials would be an adequate product for fine-dining or even Michelin-starred restaurants: "this is NOMA [one of the most renowned restaurants in the world], this is star-restaurant" (Chef 2). All chefs agreed that in restaurants with limited staff it would probably be impossible to implement the origami structures for all guests, because of the time it takes to fold the paper. However, for smaller groups of people the material could be interesting. Two chefs mentioned that it could be used for vegetarian guests (approximately $10 \%$ of diners) as a special treat. Three chefs suggested that the edible paper could be offered as a ready-made product, but four preferred to be part of the preparation process instead of using a finished product.

\subsection{Transforming}

Chefs' opinions regarding the visual cues became most apparent through the origami structures and the shape-changing reaction, and all chefs clearly showed excitement: "it moves!" (Chef 1) and "amazing" (Chef 2). Two chefs considered using the edible paper in a dish to make it melt or fall apart. Three chefs mentioned that, as a starter, the acidity could be used to start the hungry feeling, and the shape-change would immediately set the change of the diner in terms of excitement. As a main dish, two chefs suggested cannelloni that would close when sauce is added. Two chefs considered the changeable aspect of the paper, "like a present that opens up" (Chef 3 ), in a surprising desert or friandise (a small sweet which typically accompanies the coffee or tea at the end of a diner). All these ideas illustrate possible ways to implement the SCF materials, in different courses. For its changeability in combination with the sensory perception, Chef 1 suggested to develop the paper to match the organoleptic qualities (i.e. the aspects of food experienced by the senses) of the sauce. Finally, the time it takes for the paper to change shape was considered as a challenge: "You don't want the guests to wait five minutes watching the plate" (Chef 2). On the plate, shape-change should take only a few seconds; consequently, two 


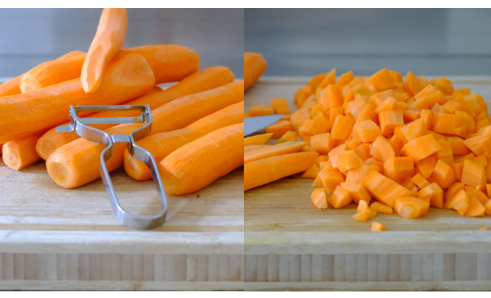

1. Peel the carrots and cut them into chunks of about $10 \mathrm{~mm}$.

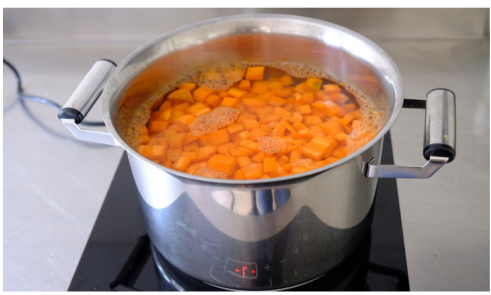

3. Bring the water to a boil and cook the carrots over medium heat until they are soft, and you can mash them with a fork.

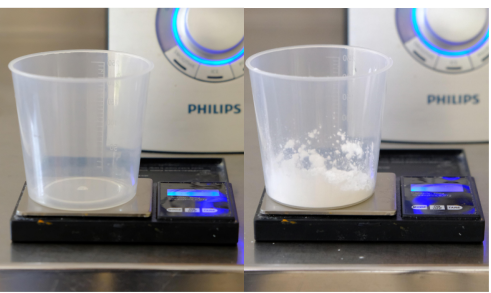

5. Dissolve the agar and CMC-Na with three tablespoons of water in a small saucepan and bring to a boil. Add the mixture, the starch, glycerol and alginate to the puree.

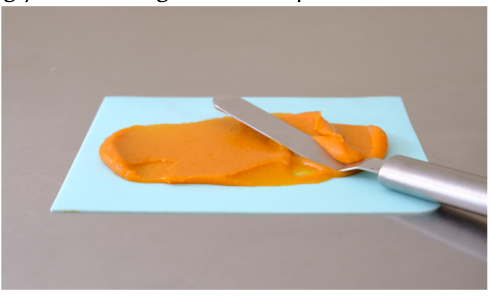

7. Place two tablespoons of the puree on a silicon sheet of about $150 \times 100 \mathrm{~mm}$.

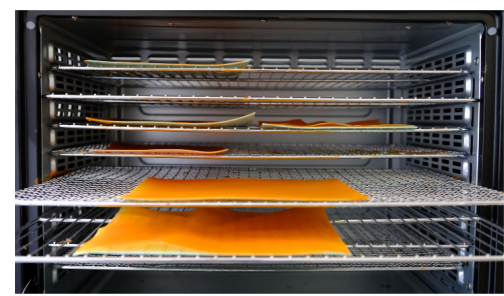

9. Dehydrate the silicone sheets in a dehydrator at $63^{\circ} \mathrm{C}$ until they are dry, depending on the thickness this should take about an hour.

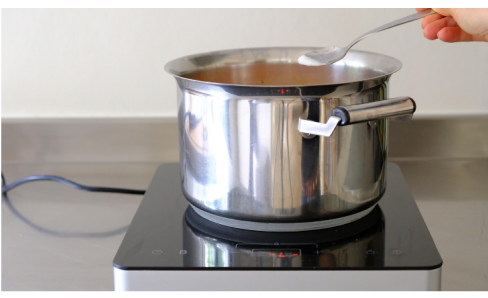

2. Place the carrot chunks in a large pan and cover them with water. Add a pinch of bakingsoda.

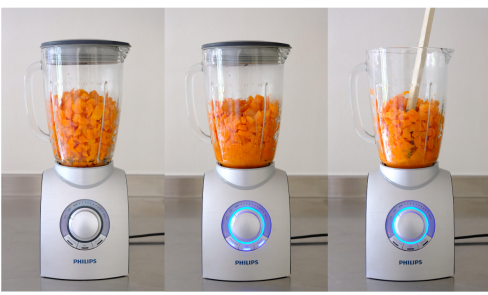

4. Use a blender to puree the carrots (we used the Philips HR2094). Steer them regularly or use the pulse function.

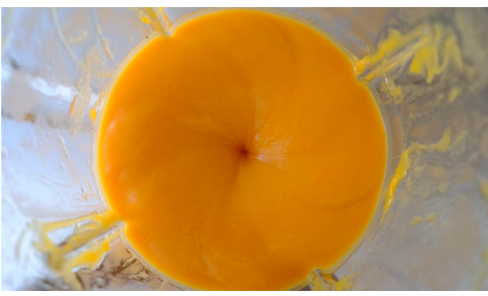

6. Continue blending the puree which should become very smooth. If necessary, use a fine mesh sieve to remove leftover lumps.

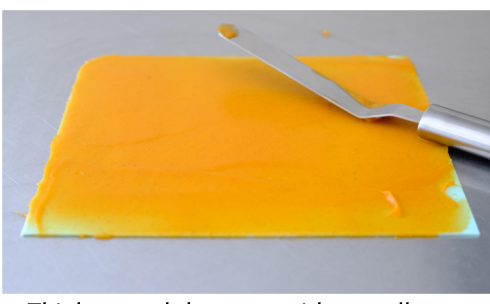

8. Thinly spread the puree with a small spreader until reaching a thickness $<1 \mathrm{~mm}$

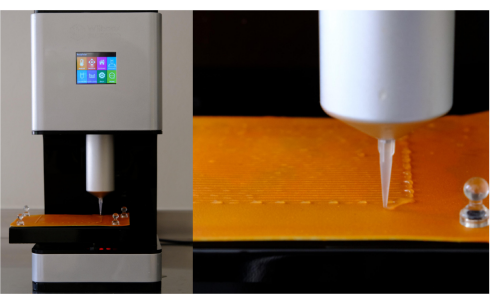

10. Print the pattern with the chitosan solution on the dehydrated sheets and let it dry. When dried print a second layer of chitosan on top of the first layer.

Figure 7: Preparation process of the shape-changing carrot paper. 


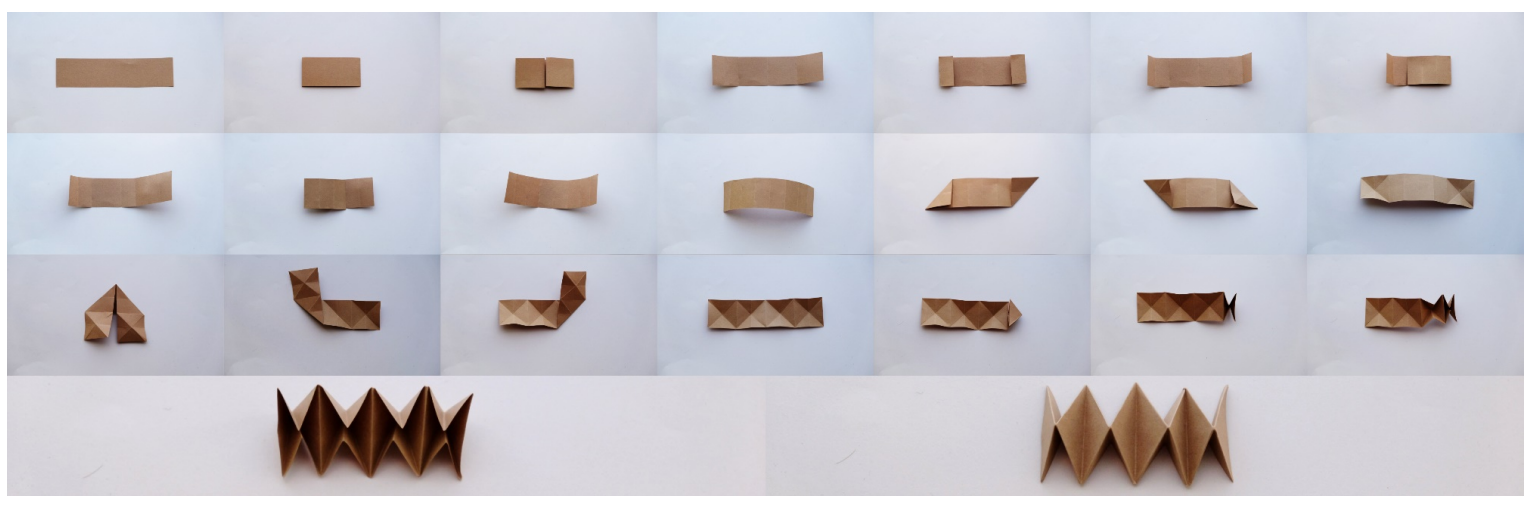

Figure 8: Folding process of an origami structure.

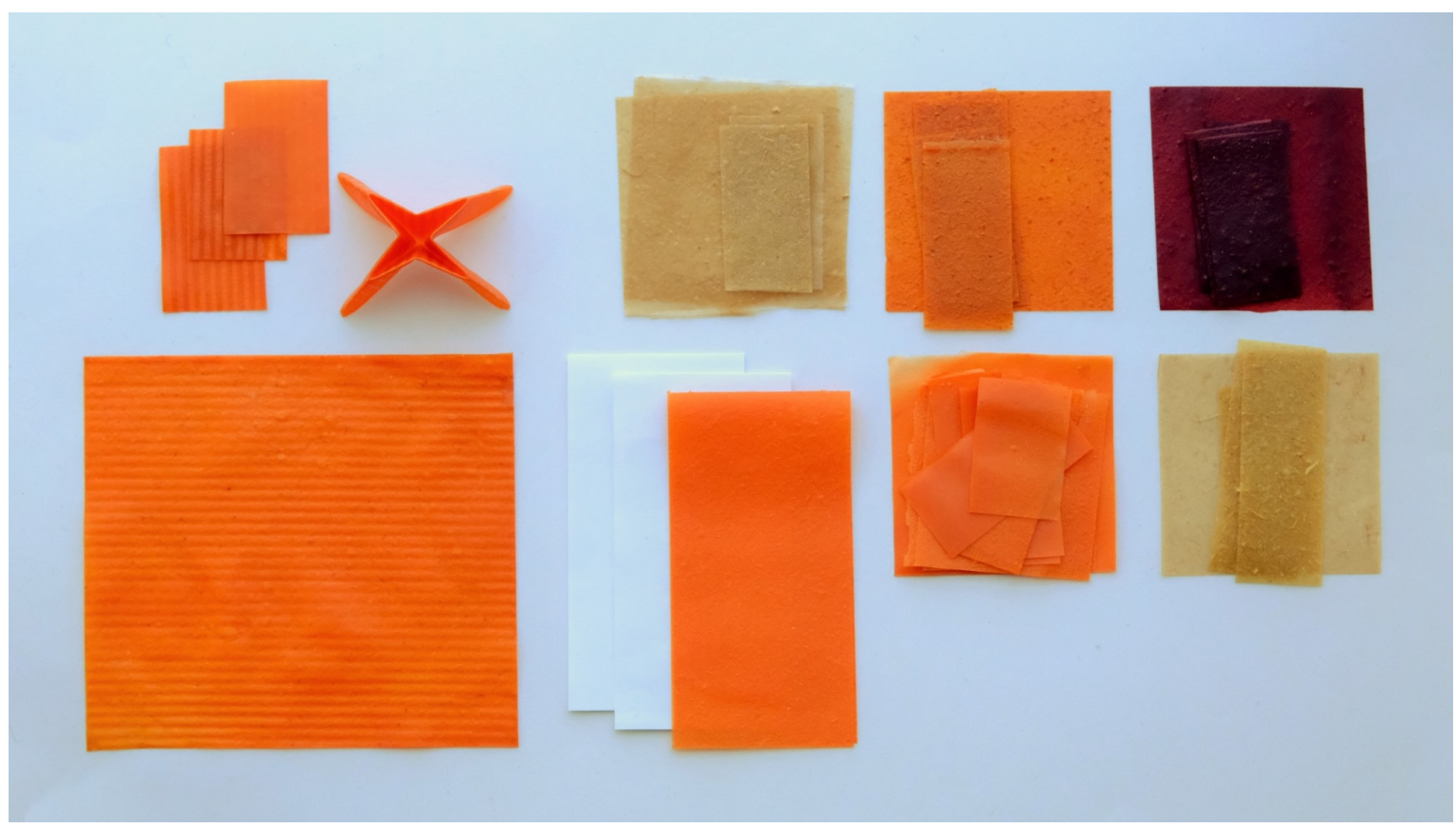

Figure 9: Composition of the material probe, from top left to bottom right: three small sheets of carrot paper with printed chitosan; one folded star structure; flavor samples of fermented kohlrabi, carrot with tomato powder, fermented plums; one large sheet of carrot paper with printed chitosan; two paper samples and a carrot sample for folding practice; flavor samples of carrot and fermented kohlrabi with mustard.

chefs considered how they could already start the transformation process before serving it.

\section{REFLECTIONS ON THE PAPER'S POTENTIAL AND APPLICABILITY IN GASTRONOMY}

\subsection{Outcomes of the Thematic Analysis}

The thematic analyses of the conversations with chefs yielded twelve themes, which we divided into four clusters (see Table 1) Texture was a recurring term, which was used in different ways and therefore related to three separate categories.
5.1.1 Cluster 1-The Paper as a Product. The first cluster addressed the qualities of the paper, seen as a product. It included quotes that referred to the ingredients that contributed to the flavor of the paper: "I think when they are fermented, they have another dimension. Much more interesting for us, flavor-wise. All the fermented, are really, really beautiful" (Chef 4). This cluster also touched upon the ingredients that contributed to the structure and functional qualities of the paper: "Texture wise is definitely the biggest deal. Because if you put too much powder in there or too many different powders, and if you put not enough powder in there, it will dissolve directly" (Chef 3). Chefs wanted to know which ingredients were used, including their ratios. They preferred to take part in 


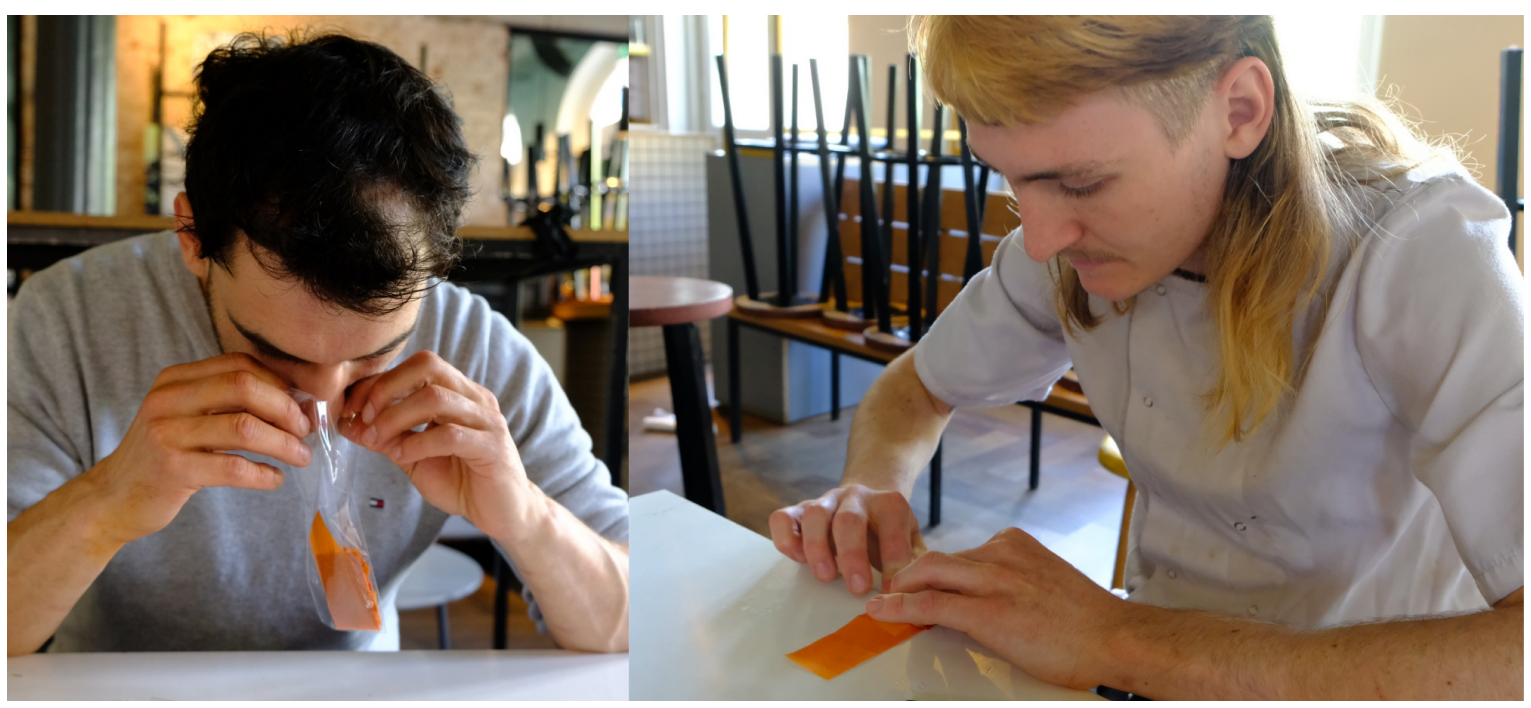

Figure 10: Two of the chefs participating in the study: smelling the paper (left) and folding the paper (right).

Table 1: Outcomes of the Thematic Analysis, defining the clusters and themes.

\begin{tabular}{ll}
\hline Clusters & Themes \\
\hline Product & Flavor ingredients \\
& Functional ingredients \\
Preparing and cooking & Preparation Process \\
& Folding Process (texture) \\
Multisensorial Experiences & Taste \\
& Visual \\
& Mouthfeel (texture) \\
Interactive Eating Experiences & Smell \\
& Dynamics \\
& Experiential (texture) \\
& Actors \\
& Overall transition \\
\hline
\end{tabular}

the making process of the paper instead of working with a ready to use material: "I think that is also a really nice thing, that you bring something kind of incomplete, that also gives us space to try, experiment. If you would just bring something that was just ready, it would be still amazing, but then you are just like a seller" (Chef 2). Further development of the material needs to consider how chefs can use the ingredients and process steps to support them in thinking creatively about alternative ingredients, but also to indicate if they are familiar (and positive) to use these ingredients in their kitchen.

5.1.2 Cluster 2 - Preparing the Paper and Cooking with it. The second cluster included quotes that referred to the process of preparing the paper: "If you make the puree from the carrots itself and you want to keep the carrot flavor alive, you can freeze dry the carrots, powder them, and use that on the paper to give it a bit more boost" (Chef 3). It also included thoughts on the folding process as experienced by the chefs, e.g., about the texture of the different types of papers as it felt when being touched: "So if it was thicker it would be kind of helpful to finish it, but now, I can't do it" (Chef 2 ). The first two clusters can be considered as part of the chef's experience while preparing their dish, or the overall recipe including ingredients and preparation process. Like the first cluster, it was important for the chefs to understand the preparation process of the material. During the participatory session, they came up with suggestions from processes they were familiar with, but also clearly indicated when certain processes were too complex or unclear. To enable chefs to develop the material themselves, further research is needed on how to reduce the complexity of the process. Alternatively, workshops can be provided so chefs can learn how to implement the process in their kitchen.

5.1.3 Cluster 3 - The Paper in a Multi-sensory Experience. The third cluster included quotes that addressed the multisensorial experiences of the edible paper, which could be considered being part of the guest experience when preparing a meal. It addressed 
quotes that related to the taste of the paper: "I don't mind that (chewiness, texture) too much because the flavor is, I think, much more intense than this one" (Chef 1); the visual experience: "How did the color become so dark?" (Chef 2); the mouthfeel, which could also be categorized as texture but when felt inside the mouth: "If the texture is providing you a little bit of discomfort, then you don't really focus on the flavor" (Chef 3); and the smell of the paper: "It smells like seaweed" (Chef 1). Consequently, all senses were considered to be important when shaping a dining experience. However, it depended per chef which senses were most important to evaluate the food. Future work should evaluate how all senses can be considered in the evaluation of our paper. Chefs can advise on how to improve these sensory experiences: "you sprayed it with something that people associate with carrots" (Chef 1).

5.1.4 Cluster 4 - The Interactive Potential of our Paper. The fourth and final cluster addressed the dish eco-system, or the inclusion of the edible paper into an envisioned guest experience. Chefs reflected on the transformative qualities of the paper, e.g., the timing of the movement: "You don't want the guests to just wait for five minutes, watching the plate like that" (Chef 2). They also discussed the idea of experiencing a texture as it changes during consumption: "If you had something nice like the langoustine, and you would pour that over the circular piece of paper, it would sort of break away" (Chef 1). Chefs also talked about the actors involved in delivering the interactive experience, including the chefs in the kitchen, the chef or waiter that would serve the dish, and the guest: "When the people come, then you will put the juice on top and then it will fold open, yes that would be funny" (Chef 3). Finally, they considered the overall transition from the kitchen to the mouth (including plating, serving and activating): "dessert wise, you can maybe just make like a small sort of knicker of caramel with nougat inside [...] if you put the fluid on top, it opens up and you have a really beautiful thing" (Chef 3 ). These examples illustrate chefs can imagine shaping new experiences with the edible paper.

\subsection{Design Implications}

While the chefs highly appreciated the engagement with the paper, further development is required before SCF materials can actually be implemented in their restaurants. Chefs perceive the food and process differently, depending on the senses they value when tasting the SCF materials and depending on how familiar they are with the processes used to create the edible paper. It is important that in the development of the paper all senses are taken into account. Examples of such improvements regard the visibility of fold lines, the mouthfeel of the paper and the flavor. Chefs already suggested roasting (instead of cooking) vegetables like tomatoes, carrots or onions to improve the taste. Also, chefs should be enabled to adjust the material with their preferred ingredients and use multiple ingredients.

In addition to enabling diversity of ingredients, the reactivity of the edible paper should also be developed further. A piece of paper coated in lemon-juice rolls-up like a cannolo within a minute. However, an origami cube can take between 3 and 15 minutes to open. If the reaction takes too long, chefs suggested that it can already be activated before serving it to the guests. However, for an interaction to be planned around the reaction, it is important that the process is constant, so the waiter knows exactly when to bring the dish to the table. From our material research, we learned that this reaction not only depends on the chitosan, but also on the type of paper, the layer thickness and $\mathrm{pH}$ of the sauce and the coding of the 3D-printer. Therefore, further research is needed to define the reactivity parameters and their interrelation, to provide chefs with more control on how to leverage it optimally.

Finally, some chefs already showed an interest in printing the chitosan onto the paper. However, alternatives (e.g., molding) could make the technology applicable to a larger audience without the need of large investments. The process should be easy to understand for chefs and they should be enabled to go through the entire process of preparing the material and implementing the material in their kitchens. Further work should simplify the process, to enable chefs in creating innovative, interactive experiences which will inspire new research directions for development in restaurant settings.

To summarize, the following guidelines should be considered when aiming to incorporate SCF into everyday culinary practices.

- Chefs should be able to develop the SCF-material according to their wishes: it should be possible to alternate the material to their preferred flavor, smell, mouthfeel, texture and color.

- Chefs should be able to produce the SCF-material themselves in their restaurant kitchen: the preparation and implementation process should be easy to understand, and it should be possible to use equipment developed for restaurant kitchens (rather than equipment developed for a lab setting).

- The shape-transforming aspect of the material should be understandable for chefs, this is needed to enable them to create new dining experiences and to shape (e.g., cut or fold) the material according to their wishes.

- SCF-materials should be optimized on their reactivity, a rapid (only a few seconds) and consistent transformation is needed when serving dishes to the guests.

\section{DISCUSSION}

Our project demonstrates how professional chefs can incorporate novel SCF materials, involving 3D printing technology, into their culinary practice. We were inspired by Wang et al. [30] and Zoran at al. [18] and adapted their ideas, which required a high-tech lab equipment, to a kitchen context. We developed a material that can be created in a professional kitchen, by the chef. In participatory sessions to evaluate our material, chefs were able to sensitize themselves to the process and material and could share their opinions on all sections of the material experience (tasting, folding and transforming). This extensive focus throughout the project lead to possible ways of implementation and development which were not considered before. Small suggestions by the chefs stimulated speculation even further regarding possible implementation, resulting in new potential interactions with the material. This provided useful directions for future research, going towards a direct and even more exciting application by chefs in their professional practice. Taking a participatory and lightweight approach, we supported chefs in shaping their creative process towards a new level of dining experiences instead of implementing a ready to use material or only envisioning futuristic dining. 
The material research followed an iterative research-throughdesign approach. After each experiment the results were evaluated, compared and subsequent steps were formulated. The participatory session with the target group, professional chefs, was conducted with the developed material probe, in which the chefs were guided through a process of tasting, folding, and transforming the material. Chefs were not informed about the fact that the material could transform beforehand. Through our approach they got to understand the process and were able to imagine how they would use the materials themselves and they provided us with possible directions for implementation and further development. Previous projects which were executed in a lab environment $[16,18,28,30,34]$ did not support the ability for chefs to implement their creativity in the process of making the material and use it in a unique way in their restaurant. Such an approach, involving more visionary materials, could possibly lead to alternative and more future-oriented speculations, but may have also overwhelmed chefs. We believe that offering a material which chefs could relate to and the lightweight process increased the appreciation by chefs and provided better feasibility and viability arguments.

In our participatory sessions, we observed that chefs were interested in the transformative properties of our material. The fact that it changed shape over time, potentially once already served, seemed to trigger their creative response. We thus began to speculate how ours and similar materials might unlock possibilities for novel, increasingly interesting and surprising forms of dining. As our findings begin to indicate, providing chefs with SCF materials might inspire them to envision dishes where certain components are interactable [14] in other ways than the ordinary (eating, smelling, cutting etc.). SCFs afford novel ways of interacting with food where, by combining and experimenting with ingredients, diners can trigger dynamic transformations that unfold in front of them. Being able to craft dishes that are dynamic and react to the diners' actions might enable chefs to explore new avenues in gastronomy design-ones that will likely surprise the diner not only through the senses (sight, smell, taste) but through interaction. That will, in turn, facilitate a move towards gastronomy design directions that are increasingly playful and interactive, which have recently been called for both in HFI $[3,4,19]$ and in food design [2, 31].

Our study did not explicitly seek to explore the interactive potential of SCF materials. Yet, considering our findings, in future work we will investigate how those properties might be leveraged by chefs to rethink their culinary practice in increasingly playful directions. We will translate our early speculations into actual food designs, supporting chefs to appropriate our material and exploring the kinds of creative uses they make of it. We will also work towards enhancing the transformative qualities of our material: our participants made it clear that the current version falls short to deliver an experience that is truly impactful, as it takes too long for the material to transform. That might often be at odds with the idea of a dynamic, interactive, and compelling play experience. In future research, we will investigate how to improve the shape-changing qualities of our material to ensure a more dynamic transformation. Despite the limitations of the current version of our transformative paper, we are excited about how our research hints at directions for food materials research that holds promise of enriching dining experiences from the perspective of interactivity and playfulness.
Having defined the guidelines for incorporating SCFs into everyday culinary practice, we see our work as the first of a series of steps that HFI researchers can take towards understanding how they can empower chefs and other food designers to craft increasingly multi-sensorial and interactive food experiences. We suggest three future directions for research in this space, which we present as provocations for other researchers interested in shape-changing materials and HFI innovation. First, our research presents a process for developing a SCF material that can be implemented by chefs in a regular kitchen setting, with the only addition of a 3D food printer. Though it is much more actionable than previous works in this space $[28,30]$, the process we present is still quite complex and might not be ideal when it comes to integrating it into chefs' existing practices and production processes. Future work should use multi-stakeholder innovation methods to explore how to carefully adjust the process of producing SCFs to the idiosyncrasies of the commonplace production processes of chefs and restaurants. Second, in our study we engaged chefs to investigate their perspectives on our material, but we never explored in-depth what might be the impact of a SCF material on the diner's experience of a meal. We suggest that, inspired by recent works in Multisensory HFI research (e.g., [21, 26, 29]), future explorations should involve cross-modal psychology methods to investigate what might be the impact of SCF materials on diners' taste perception, or on their perception of the meal experience as a whole. Finally, in this paper we began to speculate about the potential of SCF materials in terms of enriching dining experiences in interactive ways. The material proposed here, though limited, transforms dynamically once served, and affords the possibility of interacting with it. We suggest that future research should further explore the potential of augmenting food materials-not only cutlery and plate ware, but actual edible things-with interactive properties. That would present food designers with a broader palette of possibilities to design increasingly playful and interactive food experiences.

\section{CONCLUSION}

The aim of this study was to investigate how emerging, shapechanging food materials can be made accessible to chefs and diners, and why. We built on existing works in digital gastronomy and Human-Food Interaction research to propose a chef-friendly process for producing shape-changing food materials. We explored chefs' perspectives on the developed materials to better understand how they might want to integrate them into their culinary practice. We contribute a novel shape-changing food material that can be produced with the only addition of a commercial 3D food printer based on edible paper in combination with $\mathrm{pH}$-reactive chitosan and show chefs' perspectives on said material and its culinary potential. We provide guidelines on how to incorporate SCFs into everyday culinary practices. Finally, we speculate how the material could enable increasingly multi-sensory and interactive food experiences, setting new directions for human-food interaction. We hope that our work begins to hint at the potential of building stronger bridges between HFI research and the work of chefs and other culinary practitioners. 


\section{REFERENCES}

[1] Ferran Altarriba Bertran, Samvid Jhaveri, Rosa Lutz, Katherine Isbister, and Danielle Wilde. 2019. Making Sense of Human-Food Interaction. In Proceedings of the 2019 CHI Conference on Human Factors in Computing Systems (CHI '19). Association for Computing Machinery, New York, NY, USA, Paper 678, 1-13. DOI:https://doi.org/10.1145/3290605.3300908

[2] Ferran Altarriba Bertran, Rosa Lutz and Katherine Isbister. forthcoming. Where Interaction Design Meets Gastronomy: Crafting Increasingly Playful and Inter active Eating Experiences. In Proceedings of the 2nd International Conference on Food Design and Food Studies, Experiencing Food, Designing Sustainable and Social Practices (EFOOD'19). November 28-30, 2019, Lisbon, Portugal. (pre-press copy available)

[3] Ferran Altarriba Bertran and Danielle Wilde. 2018. Playing with food: reconfiguring the gastronomic experience through play. In Experiencing Food, Designing Dialogues: Proceedings of the 1st International Conference on Food Design and Food Studies (EFOOD 2017), Lisbon, Portugal. DOI:http://doi.org/10.1201/ 9781351271967-1.

[4] Ferran Altarriba Bertran, Danielle Wilde, Ernő Berezvay, and Katherine Isbister. 2019. Playful Human-Food Interaction Research: State of the Art and Future Directions. In Proceedings of the Annual Symposium on Computer-Human Interaction in Play (CHI PLAY '19). Association for Computing Machinery, New York, NY, USA, 225-237. DOI:https://doi.org/10.1145/3311350.3347155

[5] Rohit Ashok Khot and Florian Mueller. 2019. Human-Food Interaction. In Foundations and Trends Human-Computer Interaction 12, 4 (2019), 238-415. DOI: doi.org/10.1561/1100000074

[6] Alex Avila, Spencer P. Magleby, Robert J. Lang, and Larry L. Howell. 2019. Origami fold states: concept and design tool. Mechanical Sciences, 10, 1, 91-105. DOI https://doi.org/10.5194/ms-10-91-2019

[7] André Bégin and Marie-Jose van Calsteren. 1999. Antimicrobial films produced from chitosan. In International fournal of Biological Macromolecules, 26,1, 63-67. DOI: https://doi.org/10.1016/s0141-8130(99)00064-1

[8] Virginia Braun and Victoria Clarke. 2006. Using thematic analysis in psychology. In Qualitative research in psychology. 3, 2, 77-101. DOI:10.1191/1478088706qp063oa

[9] Jung-Hwa Choi, and Yoo Jin Choi. 2009. The expressive characteristics of origami fashion based on functionality. In Journal of the Korean Society for Clothing Industry. 11(4), 515-526.

[10] Design Swan. 2011. Every Origami: 15 Origami Inspired Product Designs | Design Swan. Retrieved on June 8, 2020 from https://www.designswan.com/archives/ every-origami-15-origami-inspired-product-designs.html.

[11] Elbulli. 2012. Albert y Ferran Adrià · Texturas. Retrieved on March 102020 from http://www.albertyferranadria.com/eng/texturas.html.

[12] Carmen Escoda Pastor. 2019. Origami as a Tool for Three-Dimensional Architectonic Thought. In Congreso Internacional de Expresión Gráfica Arquitectónica. 1554-1565. DOI: https://doi.org/10.1007/978-3-319-93749-6 129

[13] Jens Emil Grønbæk, Majken Kirkegaard Rasmussen, Kim Halskov, and Marianne Graves Petersen. 2020. KirigamiTable: Designing for Proxemic Transitions with a Shape-Changing Tabletop. In Proceedings of the 2020 CHI Conference on Human Factors in Computing Systems (CHI '20). Association for Computing Machinery, New York, NY, USA, 1-15. DOI:https://doi.org/10.1145/3313831.3376834

[14] J Lars-Erik Janlert and Erik Stolterman. 2016. The Meaning of Interactivity-Some Proposals for Definitions and Measures. In Human-Computer Interaction. 32,3, 103-138.

[15] Paul Jackson. 2011. Folding techniques for designers: From sheet to form. London: Laurence King Pub.

[16] Viirj Kan, Emma Vargo, Noa Machover, Hiroshi Ishii, Serena Pan, Weixuan Chen, and Yasuaki Kakehi. 2017. Organic Primitives: Synthesis and Design of pHReactive Materials using Molecular I/O for Sensing, Actuation, and Interaction. In Proceedings of the 2017 CHI Conference on Human Factors in Computing Systems (CHI '17). Association for Computing Machinery, New York, NY, USA, 989-1000. DOI:https://doi.org/10.1145/3025453.3025952

[17] Mica. 2020. Mica's Paper Craft Channels - YouTube. Retrieved on March 42020 from https://www.youtube.com/user/candyredmica401/featured.

[18] Moran Mizrahi, Amos Golan, Ariel Bezaleli Mizrahi, Rotem Gruber, Alexander Zoonder Lachnise, and Amit Zoran. 2016. Digital Gastronomy: Methods \& Recipes for Hybrid Cooking. In Proceedings of the 29th Annual Symposium on User Interface Software and Technology (UIST '16). Association for Computing Machinery, New York, NY, USA, 541-552. DOI:https://doi.org/10.1145/2984511.2984528

[19] Florian 'Floyd' Mueller, Yan Wang, Zhuying Li, Tuomas Kari, Peter Arnold, Yash Dhanpal Mehta, Jonathan Marquez, and Rohit Ashok Khot. 2020. Towards Experiencing Eating as Play. In Proceedings of the Fourteenth International Conference on Tangible, Embedded, and Embodied Interaction (TEI '20).
Association for Computing Machinery, New York, NY, USA, 239-253. DOI:https: //doi.org/10.1145/3374920.3374930

[20] Pilar Opazo. 2012. Discourse as driver of innovation in contemporary haute cuisine: The case of elBulli restaurant. In International fournal of Gastronomy

and Food Science. 1,2, 82-89. DOI: https://doi.org/10.1016/j.ijgfs.2013.06.001
[21] Betina Piqueras-Fiszman, Jorge Alcaide, Elena Roura, and Charles Spence. 2012. Is it the plate or is it the food? Assessing the influence of the color (black or white) and shape of the plate on the perception of the food placed on it. In Food Quality and Preference 24, 1, 205-208. DOI: https://doi.org/10.1016/j.foodqual.2011.08.011.

[22] Yvonne Rogers. 2011. Interaction design gone wild: striving for wild theory. interactions 18, 4 (July + August 2011), 58-62. DOI:https://doi.org/10.1145/1978822. 1978834

[23] Rayees Ahmad Shiekh, Maqsood Ahmad Malik, Shaeel A. Althabaiti and Muneer Ahmad Shiekh 2013. Chitosan as a novel edible coating for fresh fruits. In Food Science and Technology Research, 19, 2, 139-155. DOI: https://doi.org/10.3136/FSTR. 19.139

[24] Charles Spence. 2017. Gastrophysics: The new science of eating. Penguin UK.

[25] Scott E. Stapleton, Dorit Kaufmann, Helga Krieger, Jan Schenk, Thomas Gries, David Schmelzeisen. 2019. Finite element modeling to predict the steady-state structural behavior of 4D textiles. Textile Research fournal. 89,17,3484-3498. DOI: https://doi.org/10.1177/0040517518811948

[26] Nanette Stroebele and John M. De Castro. 2004. Effect of ambience on food intake and food choice. In Nutrition 20, 9, 821-838. DOI: https://doi.org/10.1016/j.nut. 2004.05.012

[27] Amelia Sydney Gladman, Elisabetta A. Matsumoto, Ralph Nuzzo, Lakshminarayanan Mahadeva, Jennifer A. Lewis. 2019. Biomimetic 4D printing. In Nature Materials 15, 413-418 DOI:https://doi.org/10.1038/nmat4544

[28] Ye Tao, Youngwook Do, Humphrey Yang, Yi-Chin Lee, Guanyun Wang, Catherine Mondoa, Jianxun Cui, Wen Wang, and Lining Yao. 2019. Morphlour: Personalized Flour-based Morphing Food Induced by Dehydration or Hydration Method. In Proceedings of the 32nd Annual ACM Symposium on User Interface Software and Technology (UIST '19). Association for Computing Machinery, New York, NY, USA, 329-340. DOI:https://doi.org/10.1145/3332165.3347949

[29] Harrar Vanessa, Charles Spence. 2013. The taste of cutlery: how the taste of food is affected by the weight, size, shape, and colour of the cutlery used to eat it. In Flavour, 1-13. DOI: https://doi.org/10.1186/2044-7248-2-21

[30] Wen Wang, Lining Yao, Teng Zhang, Chin-Yi Cheng, Daniel Levine, and Hiroshi Ishii. 2017. Transformative Appetite: Shape-Changing Food Transforms from 2D to 3D by Water Interaction through Cooking. In Proceedings of the 2017 CHI Conference on Human Factors in Computing Systems (CHI '17). Association for Computing Machinery, New York, NY, USA, 6123-6132. DOI:https://doi.org/10. 1145/3025453.3026019

[31] Danielle Wilde and Ferran Altarriba Bertran. 2019. Participatory Research through Gastronomy Design: A designerly move towards more playful gastronomy. In International fournal of Food Design, 4,1, 3-37. DOI: https://doi.org/ 10.1386/ijfd.4.1.3_1

[32] Wang Xuerong, Quan Jianquan and Chen Zongdao. 2003. Preparation of Edible Carrot Paper. In Food and Fermentation Industries, 3.

[33] Kang-De Yao, Tao Peng, Mattheus Goosen, Ji Mei Min and Yu Yin He. 1993. $\mathrm{pH}$-sensitivity of hydrogels based on complex forming chitosan: Polyether interpenetrating polymer network. In fournal of Applied Polymer Science, 48,2, 343-354. DOI: https://doi.org/10.1002/app.1993.070480218

[34] Lining Yao, Jifei Ou, Chin-Yi Cheng, Helene Steiner, Wen Wang, Guanyun Wang, and Hiroshi Ishii. 2015. BioLogic: Natto Cells as Nanoactuators for Shape Changing Interfaces. In Proceedings of the 33rd Annual ACM Conference on Human Factors in Computing Systems (CHI '15). Association for Computing Machinery, New York, NY, USA, 1-10. DOI:https://doi.org/10.1145/2702123.2702611

[35] Clement Zheng, HyunJoo Oh, Laura Devendorf, and Ellen Yi-Luen Do. 2019. Sensing Kirigami. In Proceedings of the 2019 on Designing Interactive Systems Conference (DIS '19). Association for Computing Machinery, New York, NY, USA, 921-934. DOI:https://doi.org/10.1145/3322276.3323689

[36] John Zimmerman, Jodi Forlizzi, and Shelley Evenson. 2007. Research through design as a method for interaction design research in HCI. In Proceedings of the SIGCHI Conference on Human Factors in Computing Systems (CHI '07). Association for Computing Machinery, New York, NY, USA, 493-502. DOI:https://doi.org/10. 1145/1240624.1240704

[37] Amit Zoran and Dror Cohen. 2018. Digital Konditorei: Programmable Taste Structures using a Modular Mold. In Proceedings of the 2018 CHI Conference on Human Factors in Computing Systems. Association for Computing Machinery, New York, NY, USA, Paper 400, 1-9. DOI:https://doi.org/10.1145/3173574.3173974

[38] Amit Zoran, Emiliano Arencibia Gonzalez, and Ariel Bezaleli Mizrahi. 2021. Cooking with computers: the vision of digital gastronomy. In Gastronomy and Food Science (pp. 35-53). Academic Press. 